\title{
Interactive Design Space Exploration and Optimization for CAD Models
}

\author{
ADRIANA SCHULZ, JIE XU, and BO ZHU, Massachusetts Institute of Technology \\ CHANGXI ZHENG and EITAN GRINSPUN, Columbia University \\ WOJCIECH MATUSIK, Massachusetts Institute of Technology
}

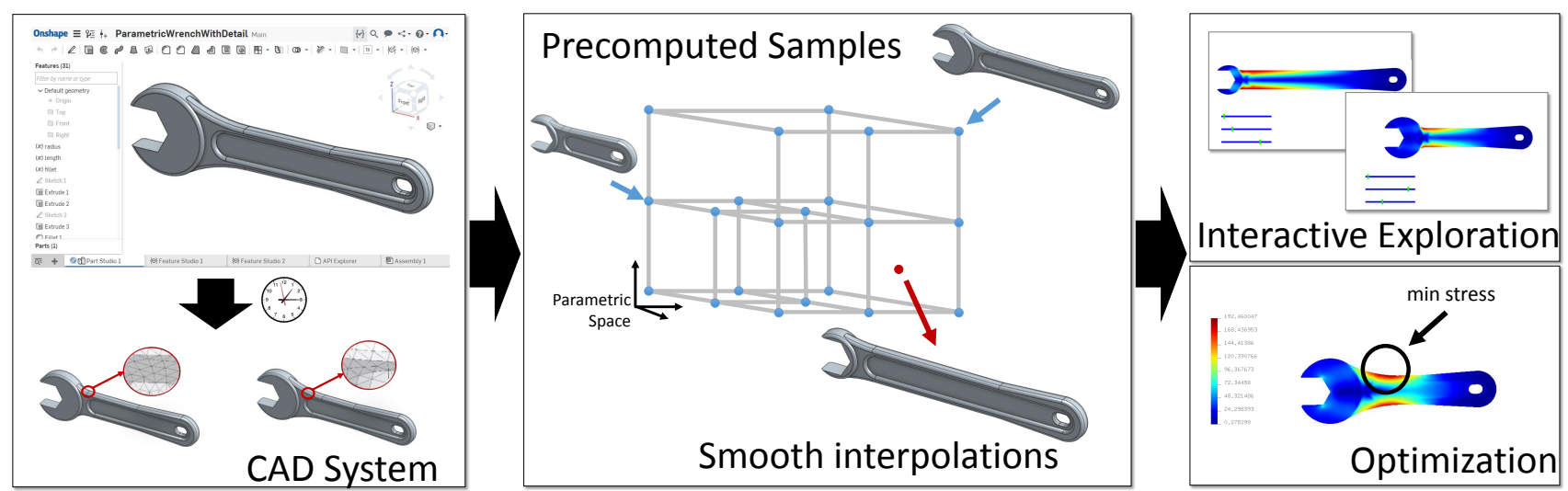

Fig. 1. Our method harnesses data from CAD systems, which are parametric from construction and capture the engineer's design intent, but require long regeneration times and output meshes with different combinatorics. We sample the parametric space in an adaptive grid and propose techniques to smoothly interpolate this data. We show how this can be used for shape optimization and to drive interactive exploration tools that allow designers to visualize the shape space while geometry and physical properties are updated in real time.

Computer Aided Design (CAD) is a multi-billion dollar industry used by almost every mechanical engineer in the world to create practically every existing manufactured shape. CAD models are not only widely available but also extremely useful in the growing field of fabrication-oriented design because they are parametric by construction and capture the engineer's design intent, including manufacturability. Harnessing this data, however, is challenging, because generating the geometry for a given parameter value requires time-consuming computations. Furthermore, the resulting meshes have different combinatorics, making the mesh data inherently discontinuous with respect to parameter adjustments. In our work, we address these challenges and develop tools that allow interactive exploration and optimization of parametric $\mathrm{CAD}$ data. To achieve interactive rates, we use precomputation on an adaptively sampled grid and propose a novel scheme for interpolating in this domain where each sample is a mesh with different combinatorics. Specifically, we extract partial correspondences from CAD representations for local mesh morphing and propose a novel interpolation method for adaptive grids that is both continuous/smooth and local (i.e., the influence of each sample is constrained to the local regions where mesh morphing can be computed). We show examples of how our method can be used to interactively visualize and optimize objects with a variety of physical properties.

This work is supported by the National Science Foundation, under grant CMMI-1547154, grant CCF-1138967, grant CMMI-1644558 and grant IIS-1409286.

Author's addresses: A. Schulz, 77 Massachusetts Ave, MIT 32-D414, Cambridge, MA 02139; email: adschulz@mit.edu.

Permission to make digital or hard copies of all or part of this work for personal or classroom use is granted without fee provided that copies are not made or distributed for profit or commercial advantage and that copies bear this notice and the full citation on the first page. Copyrights for components of this work owned by others than ACM must be honored. Abstracting with credit is permitted. To copy otherwise, or republish, to post on servers or to redistribute to lists, requires prior specific permission and/or a fee. Request permissions from permissions@acm.org.

(๑) 2017 Association for Computing Machinery.

0730-0301/2017/7-ART157 \$15.00

https://doi.org/10.1145/3072959.3073688
CCS Concepts: • Computing methodologies $\rightarrow$ Shape modeling; Shape analysis; Modeling and simulation;

Additional Key Words and Phrases: CAD, parametric shapes, simulation, precomputations, interpolation

\section{ACM Reference format:}

Adriana Schulz, Jie Xu, Bo Zhu, Changxi Zheng, Eitan Grinspun, and Wojciech Matusik. 2017. Interactive Design Space Exploration and Optimization for CAD Models. ACM Trans. Graph. 36, 4, Article 157 (July 2017), 14 pages. https://doi.org/10.1145/3072959.3073688

\section{INTRODUCTION}

Computational design typically involves fast exploration of shape variations or shape optimization. This field makes extensive use of parametric models: consistent families of geometries, each described by a given point in parameter space. Parametric shapes are advantageous because they constrain the manipulations to structurepreserving (and potentially fabrication-aware) variations and also reduce the search space, making computations more efficient. These techniques have been applied to many types of parametric shapes, from mechanical objets to garments, and masonry. So far, however, not much progress has been made in efficient exploration and optimization of the world's preeminent source of parametric data: CAD.

Practically every man-made shape that exists has once been designed by a mechanical engineer using a parametric CAD tool (e.g., SolidWorks, OpenScad, Creo, Onshape), and these models are available on extensive repositories (e.g., GrabCAD and Thingiverse, Onshape's public database). CAD shapes have the advantage of being parametric from construction, and the parameters exposed by expert engineers contain specific design intent, including manufacturing 
constraints (see Figure 2). Parametric CAD allows for many independent variables but these are typically constrained for structure preserving consideration, the need to interface with other parts, and manufacturing limitations. In this context, engineers typically expose a small number of variables which can be used to optimize the shape.

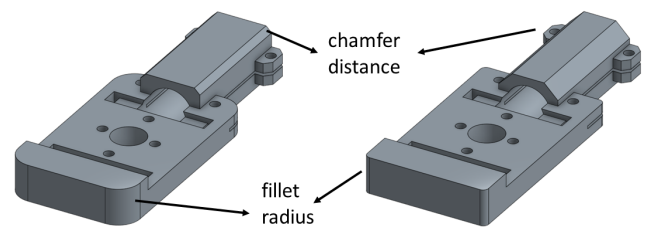

Fig. 2. When engineers design shapes, they embed their experience and knowledge in carefully selected, fabrication-aware parameters such as this fillet radius, which encapsulates a curved transition along an edge, and this chamfer distance, which describes the slope transition between two surfaces. These include fabrication limitations that take into account the different processes. This example includes minimum radius constraints on internal cuts for compatibility with milling machines.

Historically, however, it has not been a relevant concern of CAD systems to perform real-time exploration or optimization on a set of exposed parameters. It is up to the expert engineer to manually tune parameters by selecting a small set of parameter configurations to evaluate. In $\mathrm{CAD}$ systems, however, shape generation from a parameter configuration is done by recomputing the list of operations that construct the geometry. This involves computationally expensive operations (e.g., sweeping, booleans, filleting, drafting, offsetting) that can take on the order of minutes for complex models (see Figure 3). In addition, these evaluations typically involve timeconsuming physics simulations. It therefore typically takes up to a day for a mechanical engineer to select parameters for a complex shape. CAD data could greatly benefit from the progress currently being made in computer graphics in the field of fabrication-oriented design. Such tools for real-time exploration and optimization would not only make design easier for mechanical engineers but also help lower the design barrier for casual users.

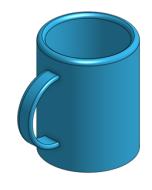

$<1$ second
3 seconds

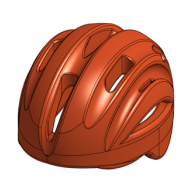

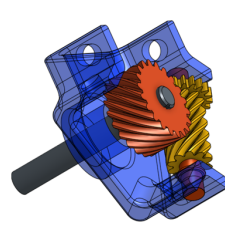

14 seconds

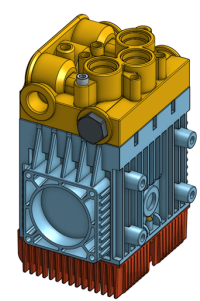

72 seconds
Fig. 3. Regeneration times for parameter changes using Onshape for models with increasing level of complexity.

To harness such CAD data, however, important open challenges must be overcome. Exploration applications require real-time evaluation of geometry, which is inherently slow for CAD parameter variations. Optimization techniques require not only shape evaluation but also the ability to evaluate derivatives. Derivatives are challenging to compute because geometric changes from parameter variations are not explicit. Furthermore, parameter changes result in different B-reps (Boundary Representation) with incomplete correspondence and generate meshes with different combinatorics, making finite-difference computations also challenging.

We address these challenges by offloading to a precomputation stage the mapping from parameters to geometry. Because different parameters can influence the geometry in different ways and to different extents, we sample the parametric domain using an adaptive grid. Interpolating in this space is challenging because each sample is a mesh with different combinatorics. Moreover, applications such as interactive exploration require continuity, and applications such as optmization require smoothness for gradient computation.

To address these problems, we propose a method that takes advantage of the underlying $\mathrm{CAD}$ representation (B-reps) to compute correspondences between shapes that are close enough in parameter space. This makes our interpolation possible, but with the caveat that, to keep the computations tractable, we must restrict the influence of each sample to local regions. But constructing an interpolation that both satisfies this locality property on a non-uniform domain and is continuous/smooth is a real challenge.

The main technical contribution of our work is a novel interpolation algorithm on adaptive grids that overcomes this difficulty, allowing for approximations across the entire parameter space that are both smooth and local. The proposed method is a generic interpolation scheme that can be applied independently of the dimensionality of the domain and can be combined with different types of basis functions (e.g. linear and cubic B-splines).

We use the precomputed data and the interpolation scheme on a system for shape exploration and optimization. Since every sample is a mesh, we can further precompute and interpolate functions defined over this mesh-for example, solutions of PDEs that describe physical phenomena.

Our interactive tool allows designers to view in real time how geometric changes impact the performance of mechanical shapes (see Figure 1). This tool is especially useful when multiple objectives are in place, and coming up with a weighting scheme for a cost function in order to perform offline optimization is challenging. This interface also allows users to understand (and potentially learn or gain intuition) how different geometric parameters affect the physical properties of the shapes-something that mechanical engineers learn though training and years of experience.

Our optimization scheme also takes into account both geometry and physical properties. This is especially useful in models that have multiple-use cases, which need to be precomputed only once and can then be optimized for different objectives and constraints.

\section{RELATED WORK}

Exploration and Optimization for Computational Design. Most of the work on computational design aims at guiding users in finding shape variations that meet certain design goals. There are two main approaches for this task. The first is to use interactive systems that allow users to manually explore the shape space. These techniques are chosen in order to give more control to the users or because design objectives depend on multiple considerations. Recent works in this space include furniture design [Umetani et al. 2012], interlocking structures [Skouras et al. 2015], model airplanes [Umetani et al. 2014], garment design [Umetani et al. 2011], and robots [Megaro 
et al. 2015], to name a few. In our work, we extend these ideas to the space of parametric $\mathrm{CAD}$ models and propose techniques that allow interactive exploration on this domain.

An alternative is to use direct shape optimization. This technique require less user input and are preferred when objective functions can be easily exposed. Example of recent work on optimization include structural stability of masonry buildings [Whiting et al. 2012] or 3D printed shapes [Prévost et al. 2013], frequency spectra [Bharaj et al. 2015], structure and motion of linkages [Bächer et al. 2015], and buoyancy [Musialski et al. 2015]. As a result of the growing interest in parametric shape optimization for fabrication-oriented design, Musialski et al. [2016] proposed a framework that can be used with any parametrization approach as long as there is access to the mapping function from parameter space to geometry and its first order derivative. Our method fulfills these stated preconditions by establishing correspondences and smooth interpolations on the space of parametric $\mathrm{CAD}$ data, allowing derivative evaluations.

Computational Design of Man-made Shapes. Previous work in computational design of man-made shapes have exploited different techniques for parametric representations. A common approach is to convert a single input mesh into a parametric model using methods such as cages [Zheng et al. 2011], linear blend skinning [Jacobson et al. 2011] or manifold harmonics [Musialski et al. 2015] These methods generate smooth deformations which have been shown to work on shapes that are more organic and for fabrication methods such as 3D printing [Prévost et al. 2013] or water jet cutting of smooth 2D parametric curves [Bharaj et al. 2015]. For more mechanical structures such as furniture [Schulz et al. 2014] and drones [Du et al. 2016], cages techniques have been applied together with algebraic techniques to preserve discrete regular patterns [Bokeloh et al. 2012]. These, however, have been limited to very small datasets, with highly constrained variations (such as dimensions of wooden plates or carbon tubes) that require laborious annotations from mechanical engineers.

Because of these limitations, some recent work looked at CAD as a means to incorporate meaningful, fabrication-aware, parametric variations. Similar to our work, Koyama et al. [2015] use precomputation on the parametric domain of CAD designed connectors. However, instead of addressing interpolation in this geometric space, they sample one-dimensional physical properties where optimization is performed. Similarly, Shugrina et al. [2015] use precomputation to allow fast exploration and validation of parametric geometry. This work also does not address interpolation but instead uses very fine sampling and snaps to the closest valid vertex for preview. This allows them to bypass the challenging interpolation problem but at the expense of occasional jumps in model appearance and high storage. An alternative approach is to represent a parametric shape as a low dimensional manifold in a high dimensional feature space. This has been shown to be effective for retrieval applications [Schulz et al. 2017] but cannot be directly extended to exploration and optimization applications.

Modern Parametric CAD. Early CAD systems were based on constructive solid geometry (CSG), which defines hierarchical boolean operations on solid primitives. Modern professional CAD systems, however, use B-rep methods that start from 2D sketches and model solids with operations such as extruding, sweeping, and revolving [Stroud 2006]. A B-rep is a collection of surface elements in the boundary of the solid and is composed of a topology (faces, edges, vertices) and a geometry (surfaces, curves, points). Each topological entity has an associated geometry (e.g., a face is a bounded portion of a surface). Modern CAD systems use the feature-based modeling paradigm [Bidarra and Bronsvoort 2000], in which solids are expressed as a list of features that define instructions on how the shape is created. These system are also parametric: each feature depends on a set of numerical values or geometric relationships that control the shape of the models [Farin et al. 2002].

The geometric operations in each feature (e.g., sweeping, booleans, filleting, drafting, offsetting) are executed by geometric kernels (such as ACIS or Parasolid) that are often licensed from other companies. An important aspect of feature execution that is handled internally by CAD systems is the referencing scheme. This scheme uniquely identifies the parts of the geometry to which each feature is applied. This mechanism is what allows models to re-generate appropriately as users make changes in parameters in the feature history. Since each commercial CAD software has its own referencing scheme, which are proprietary and generally unpublished, we refer the reader to some recent academic works on referencing [Baba-Ali et al. 2009; Bidarra et al. 2005]. In our work, we use an API to extract this referencing data from the CAD software and leverage this information in order to establish correspondences between shapes.

Interpolation on Adaptive Grids. Adaptive grids have been extensively used to efficiently store non-uniform data. The challenge for interpolating in these domains comes from the hanging nodes (also called T-junctions) at the boundary between elements at different levels. Approaches to handle discontinuities around the hanging nodes include temporarily subdividing elements [Benson and Davis 2002; Kobbelt et al. 1997], constraining T-junction nodes on edges to be linearly interpolated from their neighbors on that edge [Agarwala 2007; Losasso et al. 2004; Xu et al. 2009], using penalty functions to minimize discontinuities [Setaluri et al. 2014], or introducing generalized barycentric coordinates [Floater 2015; Sukumar and Malsch 2006]. T-Splines generalize NURBS surfaces to accommodate T-vertices [Sederberg et al. 2003]. All of these approaches have their drawbacks: they either come at the expense of generating many virtual nodes and further subdivision, lose data samples, do not guarantee continuity, are hard to extend to high dimensions, or are not easily extended to smooth interpolations.

The solution for smooth interpolations is the use of hierarchical basis functions [Lee et al. 1997]. But this comes at the price of locality: the value of a point at a given cell depends on samples that lie outside the boundary of that cell. The particular nature of our problem stems from the fact that, though we require continuous interpolations for exploration applications and smooth interpolations for derivatives in optimization problems, we also have a strong restriction of locality because averaging between samples involves morphing between meshes. Our method addresses these challenges using refinable basis functions to construct interpolants in each sampled node. We describe a refinement algorithm that updates these interpolants as elements of the K-d are refined, thereby guaranteeing locality. This algorithm is simple and easy to implement in K-d trees of any 
dimension or grading. Most importantly, it can be implemented with multiple basis functions, allowing not just continuous but smooth approximations.

\section{WORKFLOW}

The workflow of our system is shown in Figure 1. In an offline phase, CAD shapes are precomputed by adaptively sampling the parametric domain. Interpolating basis functions for each sample (Section 5) and compatible meshes for interpolation (Section 6) are computed during this phase. For some applications, in addition to storing the geometry at each sample, we also precompute and store different simulation results.

For exploration, we use the typical user interface with sliders for interactively modifying shape parameters (see top-right corner of Figure 1). Both geometry and precomputed physical properties are interpolated in real time and displayed. For this application, we use a continuous interpolation function to avoid flickering when switching between regions of the adaptive grid. For optimization, we use smooth interpolations for evaluation of continuous derivatives.

\section{PRECOMPUTATION OVERVIEW AND NOTATIONS}

A parametric shape is defined as a function that returns a geometry for each parameter configuration $x \in \mathcal{A}$, where $\mathcal{A}$ is the feasible parameter set. Here and henceforth we assume that a sample value $p_{k}$ at $x_{k} \in \mathcal{A}$ can be evaluated by interfacing with a CAD system and consists of a tetrahedral mesh. Our method computes an approximation $P(x)$ of the parametric shape by interpolating these samples.

We use a K-d tree in parameter space for sampling and interpolation (see Figure 1). We use the term element to refer to the cells of the K-d tree. The parametric shape is sampled at every corner of every element. To each sample $x_{k}$ we associate a basis function $\psi_{k}$ and approximate the shape by interpolating the samples:

$$
P(x)=\sum_{k} p_{k} \psi_{k}(x)
$$

Definition 1 (Support). The support of each basis function $S\left(\psi_{k}\right)$ is defined as region on which it assumes non-zero values.

The challenge with computing the above sum is that $p_{k}$ are meshes with varying combinatorics. To address this, let us assume a canonical mesh $p^{l}$ for each element $e_{l}$. Then, if $x \in e_{l}$, we can compute $P^{l}(x)$ by converting each sample $x_{k}$ whose support contains $e_{l}$ to this canonical mesh $p_{k}^{l}$ :

$$
P^{l}(x)=\sum_{k, e_{l} \in S\left(\psi_{k}\right)} p_{k}^{l} \psi_{k}(x) .
$$

To keep this problem tractable, we would like to limit the support of the basis functions. To this end, we make the following definitions.

Definition 2 (Element Neighborhood). The neighborhood of an element $\mathcal{B}\left(e_{l}\right)$, is defined as the set of samples on its boundary (Figure 4).

Definition 3 (Sample Neighborhood). The neighborhood of a sample $\mathcal{N}\left(x_{k}\right)$ is defined as the set of elements incident to the sample $x_{k}$. This definition can be extended to any point $p \in \mathcal{A}$, where $\mathcal{N}(p)$ is the set of elements that contain or are incident to $p$. (Figure 4).
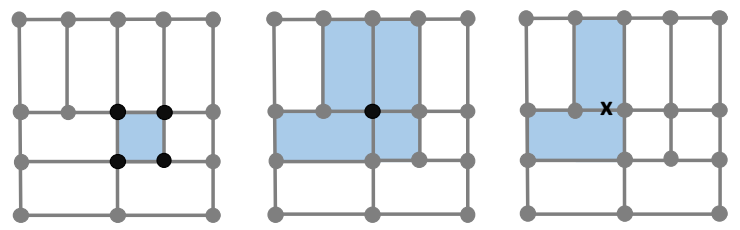

Fig. 4. The neighborhood of an element, denoted $\mathcal{B}\left(e_{l}\right)$, is defined as the set of adjacent samples (left). The neighborhood of a sample, denoted $\mathcal{N}\left(x_{k}\right)$, is defined as the set of adjacent elements (middle). We also extend the definition of $\mathcal{N}\left(x_{k}\right)$ to any point $p \in \mathcal{A}$ as the set of adjacent elements (right).

Definition 4 (Locality). We say that an approximation preserves locality if $S\left(\psi_{k}\right) \subset N\left(x_{k}\right), \forall k$. Analogously, if an approximation preserves locality, then for any sample $x_{k}$ and element $e_{l}$, then $e_{l} \subset S\left(\psi_{k}\right)$ only if $x_{k} \in \mathcal{B}\left(e_{l}\right)$

From these definitions, when an approximation preserves locality, we can rewrite equation 2 as:

$$
P^{l}(x)=\sum_{k, x_{k} \in \mathcal{B}\left(e_{l}\right)} p_{k}^{l} \psi_{k}(x) .
$$

This implies that only samples $x_{k} \in \mathcal{B}\left(e_{l}\right)$ need a mesh $p_{k}^{l}$ that is consistent with this element (Figure 5).

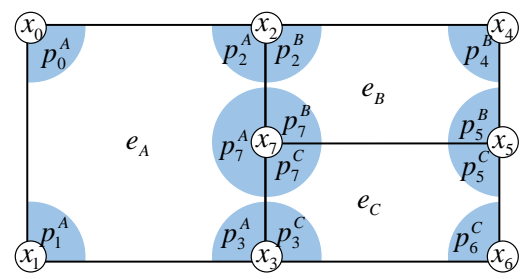

Fig. 5. When locality is enforced, the number of consistent representations $p_{k}^{l}$ that are needed at each sample $x_{k}$ depends on the cardinality of $\mathcal{N}\left(x_{k}\right)$.

In order to guarantee that there are no discontinuities when crossing between elements, we would like $P(x)$ to be continuous. From Equation 1, we see that it is enough to ensure that $\psi_{k}$ is continuous for all samples $x_{k}$.

\subsection{Refinement Relations}

To propose a scheme that guarantees locality and continuity on an adaptive grid, we take advantage of basis functions that observe a refinement relation which we define now for notation purposes. A refinement relation allows for expressing a basis function as the sum of dilated translated versions [Zorin and Schroder 2000]. In our algorithm we use linear B-splines, illustrated in Figure 6. A basis function from a coarser level can be written as a linear combination of basis functions from the next finer level:

$$
\phi_{i}^{j}(x)=\sum_{n} a_{i n}^{(j+1)} \phi_{n}^{(j+1)}(x),
$$

where the superscript $j$ indicates the level of refinement $(j=0$ corresponding to the original, coarsest grid), and the subscripts $i$ and $n$ index the basis functions at the respective levels. In the case of linear B-splines, the coefficient $a_{i n}$ do not depend on $j$ and are given by

$$
\phi_{i}^{(j)}(x)=\frac{1}{2} \phi_{(2 i-1)}^{(j+1)}(x)+\phi_{(2 i)}^{(j+1)}(x)+\frac{1}{2} \phi_{(2 i+1)}^{(j+1)}(x) .
$$




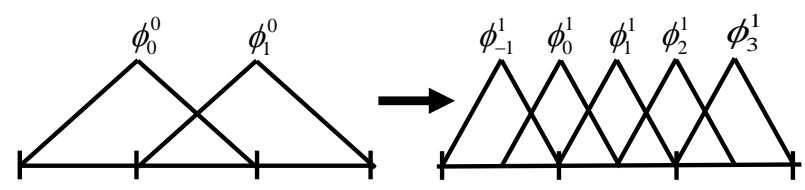

Fig. 6. Refinement of linear B-splines.

\subsection{Adaptive Sampling}

We build the K-d tree as follows. The parametric domain defines an element $e_{0}$ at the coarsest level where every corner vertex is sampled. We associate to each sample $x_{k}$ a single B-spline at the coarsest level, $\psi_{k}=\phi_{k}^{0}$.

We establish a canonical mesh $p^{l}$ for each element by evaluating the geometry at the center of the element and using the mapping algorithm (Section 6) to establish a consistent meshing inside each element. We store the consistent meshes for each of the samples on the cell boundary $p_{k}^{l}$ (see Figure 5).

We initially refine all samples once in each direction and then continue to refine adaptively. At this stage, to decide whether an element $e_{l}$ needs refinement, we evaluate the application-specific approximation error at the center of $e_{l}$ and refine it if this value is above a given threshold. Since we have consistent meshes, the error above can easily be computed with application-specific metrics (see Section 7). To decide the refinement direction on a high-dimensional $\mathrm{K}-\mathrm{d}$ tree, we compute the distance in each direction and use the maximum, again taking advantage of the consistent meshes. This process is similar to the work of Shugrina et al. [2015].

Our method iterates over the leaf nodes of the K-d tree checking which need to be refined. We use a priority queue with the size of the element as priority. When an element $e_{l}$ is split, its children are added to this queue. Also, since this refinement also affects the interpolated result on the elements adjacent to the split element $e_{l}$, these are also added to the queue.

\subsection{Refinement Notations}

When an element is refined, new samples are added along the split and the basis functions $\psi_{k}$ associated to both the new samples and the original samples on the boundary of $e_{l}$ are recomputed to ensure that locality is preserved. In our refinement method, each basis function is written as a sum of linear B-splines:

$$
\psi_{k}(x)=\sum_{i, j} \alpha_{k}^{i, j} \phi_{i}^{j}(x) .
$$

Then, our approximation can be expressed as:

$$
P(x)=\sum_{i, j}\left(\sum_{k} \alpha_{k}^{i, j} p_{k}\right) \phi_{i}^{j}(x) .
$$

We can extend the definitions above to this alternative expression. We extend the definition of support to $\phi_{i}^{j}$, where $S\left(\phi_{i}^{j}\right)$ is the region where it assumes non-zero values. Since locality was defined as $S\left(\psi_{k}\right) \subset N\left(x_{k}\right), \forall k$, an approximation preserves locality if and only if $S\left(\phi_{i}^{j}\right) \subset \mathcal{N}\left(x_{k}\right), \forall k$ such that $a_{k}^{i, j} \neq 0$. We can therefore make the following definition and remark:

Definition 5 (Local Point). We say that a point $y_{j}^{i} \in \mathcal{A}$ is a local point of a B-spline $\phi_{i}^{j}$ if $S\left(\phi_{i}^{j}\right) \subset \mathcal{N}\left(y_{j}^{i}\right)$.
Definition 6 (Local Sample Set). We define the local sample set $\mathcal{L}_{j}^{i}$ of a B-spline $\phi_{i}^{j}$ as the set of samples $x_{k}$ that are local points of $\phi_{i}^{j}$, i.e, such that $S\left(\phi_{i}^{j}\right) \subset \mathcal{N}\left(x_{k}\right)$.

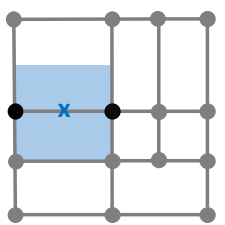

Fig. 7. A linear B-spline $\phi_{i}^{j}$ is illustrated in blue: the blue "'x"' is the center and the region where it assumes non-zero values is shaded in light blue. The local sample set of this linear B-spline $\mathcal{L}\left(\phi_{i}^{j}\right)$, is defined as the set of samples $x_{k}$ such that $S\left(\phi_{i}^{j}\right) \subset \mathcal{N}\left(x_{k}\right)$ and is illustrated in black.

Remark 1 (Locality). An approximation preserves locality if $\alpha_{k}^{i, j}=$ $0, \forall k \notin \mathcal{L}_{j}^{i}$.

These defintions are useful when defining the algorithm that ensures locality and to prove that our algorithm also ensures linear precision (linear functions are recovered exactly) and the partition of unity property (the sum of all basis functions is one).

\section{ADAPTIVE REFINEMENT STRATEGY}

We discuss our method for refining elements in a K-d tree, which guarantees both locality and continuity.

\subsection{Motivation}

As discussed in previous work, an approximation over a discretized domain can be described from two perspectives: elements and basis functions. From the element perspective, the approximation is defined over each element by an interpolation of the samples on the boundary. From the basis function perspective, the approximation is described as a sum of basis functions weighted by sampled values.

Though element representations drive refinement operations that ensure locality, they introduce discontinuities along T-junctions on high-dimensional adaptive grids. Consider for example the standard scheme, where multi-linear interpolation is done on each element. Figure 8 illustrates the discontinuities at the boundary between elements at different levels that results from this approach and are not present in our method.

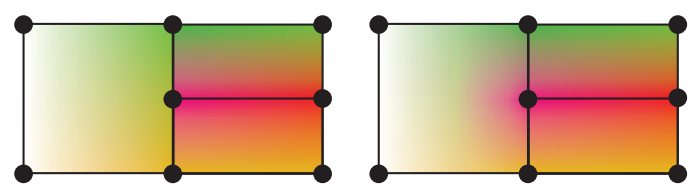

Fig. 8. Comparison between multi-linear interpolation (left), where discontinuities appear along T-junctions, and our method (right), where the interpolation is continuous.

Refinement of basis functions, on the other hand, can be used to add detail while preserving continuity. Two approaches of basis refinement have been discussed in previous work: hierarchical refinement, in which dilated basis functions are added in the center of an element [Forsey and Bartels 1988]; and quasi-hierarchical refinement, which takes advantage of refinement relations and has 

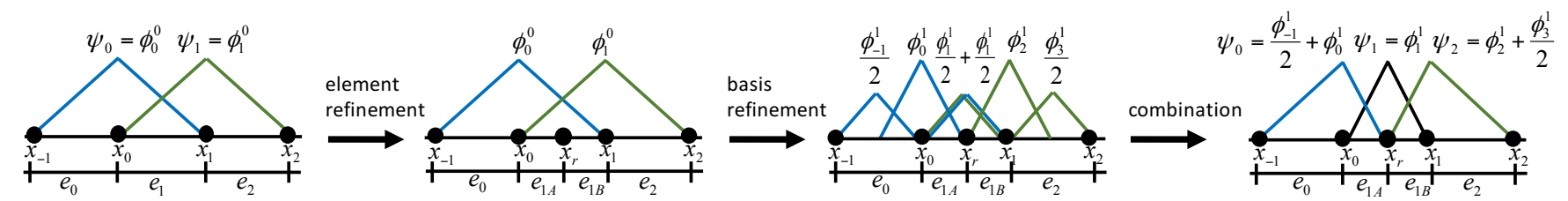

Fig. 9. Our method of combining element refinement with basis refinement: interpolated values inside each element only depend on the samples that lie on the boundary of that element.

the advantage of restricting the disparity between levels [Grinspun et al. 2002]. Both of these basis refinement methods, however, have the disadvantage of not preserving locality (see Figure 10).

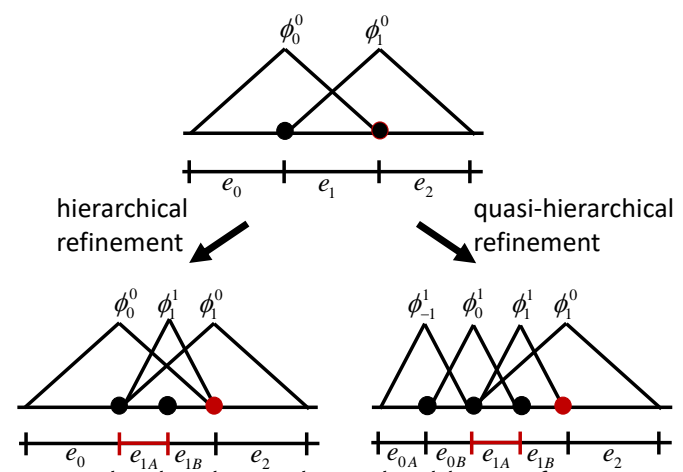

Fig. 10. Hierarchical and quasi-hierarchical basis refinement strategies. Both of these schemes violate locality on element $e_{1 A}$ since the basis function $\phi_{1}^{0}$ does not vanish on this element. The sample outside $\mathcal{B}\left(e_{1 A}\right)$ affecting this element is highlighted in red.

We propose an alternative method that combines the element and basis functions perspectives. Our algorithm does refinement based on elements to guarantee locality but uses basis functions to guarantee continuity. Drawing ideas from the quasi-hierarchical scheme for basis refinement, our algorithm takes advantage of refinement relations. Figure 9 illustrates our algorithm on a simple one-dimensional example. Initially, the approximation on element $e_{1}$ is given by:

$$
P^{1}(x)=p_{0}^{1} \underbrace{\phi_{0}^{0}(x)}_{\psi_{0}(x)}+p_{1}^{1} \underbrace{\phi_{1}^{0}(x)}_{\psi_{1}(x)} .
$$

In the first step of our method, we refine the element $e_{1}$ by adding a sample $x_{r}$ in center of $e_{1}$ and splitting $e_{1}$ into two new elements: $e_{1 A}$ and $e_{1 B}$. In the second step, we refine all basis functions that overlap $e_{1}$; we can therefore express the approximation as

$$
\begin{aligned}
P^{1}(x)= & p_{0}^{1} \underbrace{\left(\frac{1}{2} \phi_{-1}^{1}(x)+\phi_{0}^{1}(x)+\frac{1}{2} \phi_{1}^{1}(x)\right)}_{\psi_{0}(x)} \\
& +p_{1}^{1} \underbrace{\left(\frac{1}{2} \phi_{1}^{1}(x)+\phi_{2}^{1}(x)+\frac{1}{2} \phi_{3}^{1}(x)\right)}_{\psi_{1}(x)} .
\end{aligned}
$$

Finally, we define the new basis function by associating the B-splines that break locality-in this case, $\phi_{1}^{1}$-to the sample $x_{r}$. The resulting approximation is

$$
\begin{array}{r}
P^{1}(x)=p_{0}^{1} \underbrace{\left(\frac{1}{2} \phi_{-1}^{1}(x)+\phi_{0}^{1}(x)+\right)}_{\psi_{0}(x)} \\
+p_{1}^{1} \underbrace{\left(\phi_{2}^{1}(x)+\frac{1}{2} \phi_{3}^{1}(x)\right)+p_{r}^{1} \underbrace{\left(\phi_{1}^{1}(x)\right)}_{\psi_{r}(x)} .}_{\psi_{1}(x)}
\end{array}
$$

From this, we can write $P^{1 A}(x)=p_{0}^{1 A} \psi_{0}(x)+p_{r}^{1 A} \psi_{r}(x)$ and $P^{1 B}(x)=$ $p_{1}^{1 B} \psi_{1}(x)+p_{r}^{1 B} \psi_{r}(x)$, guaranteeing locality on the new elements. Since our refinement strategy only involves refining B-spline functions using the refinement relations and regrouping them into composed basis functions, partition of unity is preserved. Moreover, we observe from the two equations above that, if $p_{r}^{1}=\left(p_{0}^{1}+p_{1}^{1}\right) / 2$, then the refinement operation does not alter the solution, which indicates that linear precision is preserved. From Figure 9 we observe that this strategy is equivalent to standard element refinement in the onedimensional case, in which the hanging nodes issue does not arise. However, this strategy can be generalized to high dimensions, allowing continuous interpolation on adaptive grids that preserve locality. Furthermore, this method can be extended to higher-order basis functions, such as cubic B-splines that allow smooth interpolations (Section 5.3).

\subsection{Algorithm}

The general algorithm in high dimensions for refining an element $e_{l}$ given a split direction $d$ is described by the following steps:

step 1: Refine the element $e_{l}$.

step 2: Refine the basis functions overlapping $e_{l}$.

step 3: Redefine $\psi_{k}$ to ensure locality.

Definition 7 (Common Cuboid). Given any set of elements $e_{l}$ with non-empty intersection, we define the common cuboid as the region $R=\cap e_{l}$. Since each element is a K-dimensional cuboid, $R$ is a (possibly lower dimensional) cuboid.

In step 1 , the element $e_{l}$ is refined, creating two child elements $e_{l A}$ and $e_{l B}$ and new samples are added to guarantee that there exists a sample on the corners of every common cuboid (see Figure 11). Only intersections of $e_{l A}$ and $e_{l B}$ need to be checked and samples are added only if they do not previously exist and are generated by evaluating the $\mathrm{CAD}$ shape.

In step 2, we use the refinement relations to refine all the B-splines $\phi_{i}^{j}$ overlapping $e_{l}$ where the size of their support $S\left(\phi_{i}^{j}\right)$ is larger than the size of the element $e_{l}$ in direction $d$. Since locality is preserved in every iteration, only the B-splines $\phi_{i}^{j}$ in the terms of $\psi_{k}, x_{k} \in \mathcal{B}\left(e_{l}\right)$ (Equation 6) can overlap $e_{l}$ and therefore need to be checked. We 

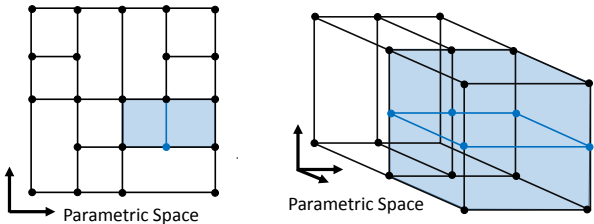

Fig. 11. Example of samples added for a given split in 2D and 3D. Split element, split plane and added samples are shown in blue.

can show that at each iteration, step 2 needs to perform at most one level of refinement (see Section S2 of supplemental material). This step updates the values of $\alpha_{k}^{i, j}$. By substituting Equation 4 into Equation 6 , the updated values, $\bar{\alpha}_{k}^{i, j}$, are:

$$
\left\{\begin{array}{l}
\bar{\alpha}_{k}^{n, j+1}=\alpha_{k}^{n, j+1}+a_{i n}^{(j+1)} \alpha_{k}^{i, j}, \forall n, k \\
\bar{\alpha}_{k}^{i, j}=0, \forall k
\end{array}\right.
$$

From the properties of refinement relations, this does not alter the summed value in Equation 7 and, therefore, the properties of partition of unity and linear precision are preserved after this step.

Finally, in step 3, we redefine the basis functions $\psi_{k}$ to enforce locality, which is done by updating the values $\alpha_{k}^{i, j}$ (see Equation 6). There are multiple assignments of these values that guarantee locality. From Remark 1, locality can be guaranteed simply by setting the coefficients that violate locality to zero $\left(\alpha_{k}^{i, j}=0, \forall x_{k} \notin \mathcal{L}_{i}^{j}\right)$. Simply zeroing out these coefficients, however, would make the resulting approximation function $P(x)$ break important interpolation properties. We therefore propose a refinement strategy that guarantees locality but also enforces a partition of unity and linear precision.

Enforcing a Partition of Unity. From Equation 7, a partition of unity is guaranteed if the updated values of $\alpha_{k}^{i, j}, \bar{\alpha}_{k}^{i, j}$, satisfy the following property for every $\phi_{i}^{j}$ :

$$
\sum_{k} \bar{\alpha}_{k}^{i, j}=\sum_{k} \alpha_{k}^{i, j}
$$

Therefore, in order to ensure both locality and a partition of unity, we first select all of the B-splines $\phi_{i}^{j}$ that violate locality (according to Remark 1) with the refinement of $e_{l}$. Since we assume that locality was preserved in previous iterations, only the B-splines $\phi_{i}^{j}$ in the terms of $\psi_{k}, x_{k} \in \mathcal{B}\left(e_{l}\right)$ (Equation 6) can overlap $e_{l}$ and therefore need to be checked. Then, for each of these B-splines, we must zero out the coefficients $\alpha_{k}^{i, j}, x_{k} \notin \mathcal{L}_{i}^{j}$ and distribute their added value amongst other coefficients $\alpha_{k}^{i, j}, x_{k} \in \mathcal{L}_{i}^{j}$ to enforce that the sum above is preserved.

To achieve this, we must show that the set $\mathcal{L}_{i}^{j}$ is not empty and define a method for finding samples in this set and using them to update $\alpha_{k}^{i, j}$.

Local Point Lemma. For every $\phi_{i}^{j}$ there exists a local point $y_{i}^{j}$.

The proof is given in the supplemental material.

Given a local point $y_{i}^{j}$, we propose the following reallocation procedure. Let $R_{i}^{j}$ be the common cuboid defined by the intersection of the elements $e_{l} \in \mathcal{N}\left(y_{i}^{j}\right)$. In Figure $7, R_{i}^{j}$ is the line segment between the two black samples. The samples $x_{r}$ on the corners of this cuboid are guaranteed to exist by step 1 .

Claim. The corner samples $x_{r} \in R_{i}^{j}$ are in $\mathcal{L}_{i}^{j}$.

Proof. Let $e_{l} \in \mathcal{N}\left(y_{i}^{j}\right)$. Then $x_{r} \in \cap e_{l}$ implies $x_{r} \in \mathcal{B}\left(e_{l}\right)$ and therefore $e_{l} \in \mathcal{N}\left(x_{r}\right)$. From this we conclude that $\mathcal{N}\left(y_{i}^{j}\right) \subset$ $\mathcal{N}\left(x_{r}\right), \forall x_{r} \in R_{i}^{j}$. Since we assume $S\left(\phi_{i}^{j}\right) \subset \mathcal{N}\left(y_{i}^{j}\right)$, from the definition of $\mathcal{L}$ it follows that $x_{r} \in \mathcal{L}_{i}^{j}, \forall x_{r} \in R_{i}^{j}$.

Since $y_{i}^{j} \in R_{i}^{j}$, we can express it as the convex combination $y_{i}^{j}=\sum \beta_{r}^{i, j} x_{r}, \sum \beta_{r}^{i, j}=1$ using multi-linear weights $\beta^{i, j} r$ where $x_{r}$ are the samples in $R_{i}^{j}$. Let $\alpha_{i}^{j}=\sum_{k} \alpha_{k}^{i, j}$. We then set

$$
\bar{\alpha}_{r}^{i, j}= \begin{cases}\alpha_{i}^{j} \beta_{r}^{i, j}, & x_{r} \in R_{i}^{j} \\ 0, & \text { otherwise. }\end{cases}
$$

Partition of unity is preserved since $\sum_{r} \beta^{i, j}=1$. This completes the realocation procedure.

It can be shown that if $c_{i}^{j}$ is the center of the B-splines $\phi_{i}^{j}$, then $c_{i}^{j}$ is a local point of $\phi_{i}^{j}$. We can therefore use the realocation procedure with $y_{i}^{j}=c_{i}^{j}$ to find a solution that guarantees locality and a partition of unity.
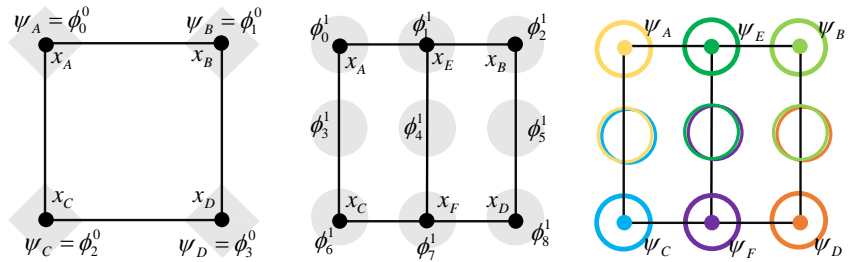

Fig. 12. Illustration of refinement in two dimensions. Letters are used to index $x_{k}$ for clarity. When the element is split, new samples are added, the $\mathrm{B}$-splines $\phi_{i}^{j}$ are refined and the new basis functions $\psi_{i}^{j}$ are recomputed to ensure locality.

A 2D example of this is shown in Figure 12. In the first step, we split the element generating samples at $x_{E}$ and $x_{F}$. In the second step, we refine all the basis function shown since they all overlap the element. In the third step we find the B-splines that violate locality (according to Remark 1). For example, the B-spline $\phi_{4}^{1}$ violates locality since $\alpha_{A}^{4,1}=\alpha_{B}^{4,1}=\alpha_{C}^{4,1}=\alpha_{D}^{4,1}=1 / 4$ and the samples $x_{A}, x_{B}, x_{C}$ and $X_{D}$ are not in $\mathcal{L}_{4}^{1}$. As discussed above, we compute $R_{4}^{1}$,which is the line segment between $x_{E}$ and $x_{F}$. We express $c_{4}^{1}$ as a function of samples at $R_{4}^{1}, c_{4}^{1}=\left(x_{E}+x_{F}\right) / 2$. We therefore we update the values of $\alpha$ setting $\bar{\alpha}_{E}^{4,1}=\bar{\alpha}_{F}^{4,1}=1 / 2$ and zero elsewhere. Similarly, we update the coefficients of $\phi_{1}^{1}$ and $\phi_{7}^{1}$, which violates locality. From this we can redefine $\psi_{E}=\phi_{1}^{1}+\phi_{4}^{1} / 2, \psi_{F}=\phi_{7}^{1}+\phi_{4}^{1} / 2$, $\psi_{A}=\phi_{0}^{1}+\phi_{3}^{1} / 2, \psi_{B}=\phi_{2}^{1}+\phi_{5}^{1} / 2, \psi_{C}=\phi_{0}^{6}+\phi_{3}^{1} / 2$, and $\psi_{D}=\phi_{8}^{1}+\phi_{5}^{1} / 2$. The colors on the right in Figure 12 indicate the influence of each sample, which are local after this redefinition.

Enforcing Linear Precision. In addition to locality and partition of unity, we would like our interpolation to exactly reproduce linear functions. We will propose a method for defining the $\bar{\alpha}_{k}^{i, j}$ such that if the evaluations function $x_{k} \longmapsto p_{k}$ is linear and linear precision 
was enforced in all previous iterations, then

$$
\sum_{k} \bar{\alpha}_{k}^{i, j} p_{k}=\sum_{k} \alpha_{k}^{i, j} p_{k}
$$

From Equation 7, this implies that, in the linear case, the approximation does not change with refinement and therefore continues to exactly reproduces linear functions after each iteration.

For intuition, let us first consider the special case when $\alpha_{i}^{j}=$ $\sum_{k} \alpha_{k}^{i, j}=1$. Under this assumption, the realocation procedure with $y_{i}^{j}=c_{i}^{j}$ results in $c_{i}^{j}=\sum_{k} \bar{\alpha}_{k}^{i, j} x_{k}$, since $\bar{\alpha}_{r}^{i, j}=\beta_{r}^{i, j}, \forall x_{r} \in R_{i}^{j}$. Let $p_{c_{i}^{j}}$ be the evaluation at $c_{i}^{j}$. If the evaluation function is linear, then above expression yields $p_{c_{i}^{j}}=\sum_{k} \bar{\alpha}_{k}^{i, j} p_{k}$. On the other hand, If linear precision was guaranteed in all previous iterations, then $p_{c_{i}^{j}}=P\left(c_{i}^{j}\right)$. Since every B-spline evaluates to 1 on its center and partition of unity is guaranteed, Equation 7 yields $P\left(c_{i}^{j}\right)=\sum_{k} \alpha_{k}^{i, j} p_{k}$. From this we conclude that Equation 11 holds and therefore linear precision is preserved.

Unfortunately, in general, we cannot guarantee that $\alpha_{i}^{j}=1$. While this was true in the example in Figure 12, this is usually not the case in high dimensions and after multiple iterations. Our solution therefore is to use the realocation procedure with $y_{i}^{j}=\sum_{k} \alpha_{k}^{i, j} x_{k} / \alpha_{i}^{j}$ (notice that when $\sum_{k} \alpha_{k}^{i, j}=1, y_{i}^{j}=c_{i}^{j}$ ). This is possible because we can prove that for this definition of $y_{i}^{j}, S\left(\phi_{i}^{j}\right) \subset \mathcal{N}\left(y_{i}^{j}\right)$ (supplemental material). By directly following the sequence of arguments above, we can show that, for this definition of $y_{i}^{j}$, linear precision is guaranteed in the general case.

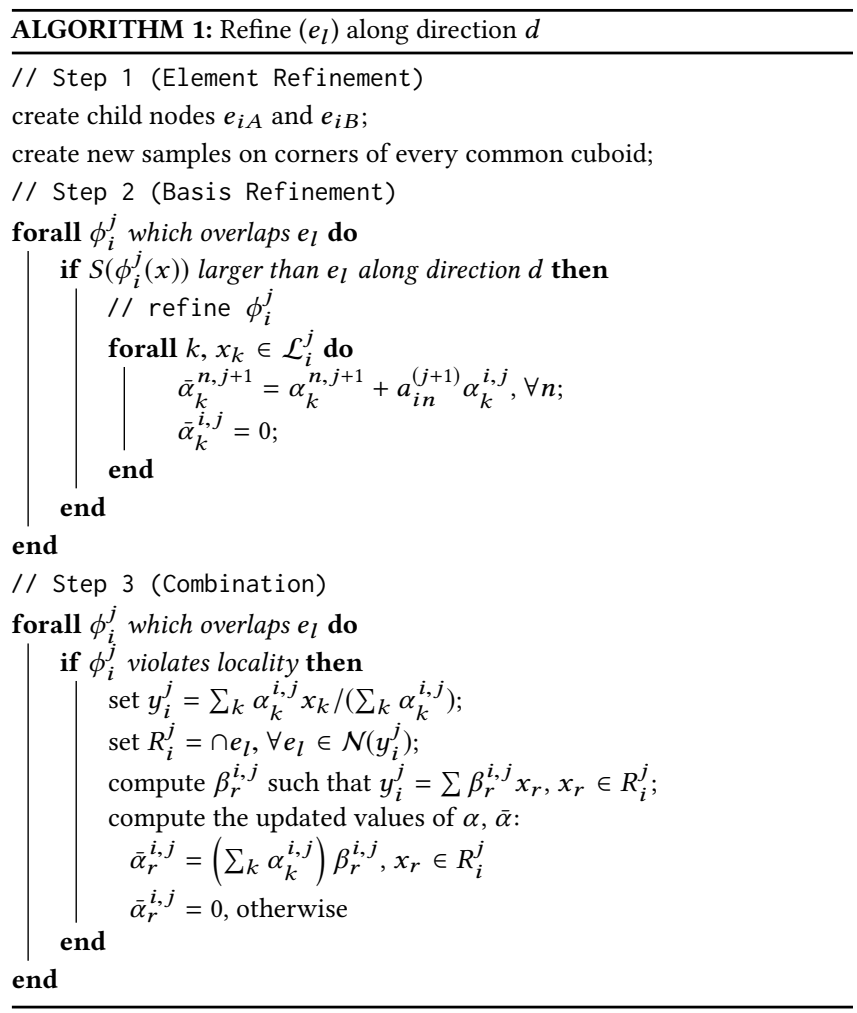

\subsection{Extension to Cubic B-splines}

To extend our method to cubic B-splines, we first redefine locality. On a uniform grid, cubic B-splines generate smooth interpolations by introducing dependencies on adjacent samples. We therefore define the neighborhood $\overline{\mathcal{N}}\left(x_{k}\right)$ of a sample $x_{k}$ as the set of elements that either contain the sample $x_{k}$ or are adjacent to some element that contains the sample $x_{k}$. Analogously, we define the neighborhood $\overline{\mathcal{B}}\left(e_{l}\right)$ of an element $e_{l}$ as the set of samples on its boundary and on the boundaries of its adjacent elements (see Figure 13).
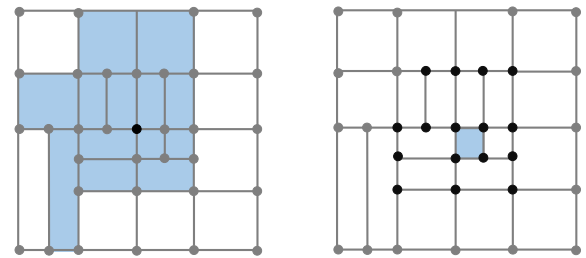

Fig. 13. The neighborhood $\overline{\mathcal{N}}\left(x_{k}\right)$ of a sample $x_{k}$ (left) and the neighborhood $\overline{\mathcal{B}}\left(e_{l}\right)$ of an element $e_{l}$ (right) for cubic B-splines.

As in the linear case, we say that an approximation preserves locality if $\forall k, S\left(\psi_{k}\right) \in \bar{N}\left(x_{k}\right)$; or, equivalently, if, for each element $e_{l}$, any sample $x_{k}$ whose associated basis function $\psi_{k}$ does not vanish on $e_{l}$ is contained in the neighborhood $\overline{\mathcal{B}}\left(e_{l}\right)$ of that element. We use the same method described in Algorithm 1, refining cubic B-splies in step 2 if their support is larger than twice the size of the refined element $e_{l}$ in direction $d$. We use following refinement relation for cubic B-splines (see Figure 14):

$$
\begin{aligned}
\phi_{i}^{(j)}(x)= & \frac{1}{8} \phi_{(2 i-2)}^{(j+1)}(x)+\frac{1}{2} \phi_{(2 i-1)}^{(j+1)}(x)+\frac{6}{8} \phi_{(2 i)}^{(j+1)}(x) \\
& +\frac{1}{2} \phi_{(2 i+1)}^{(j+1)}(x)+\frac{1}{8} \phi_{(2 i+2)}^{(j+1)}(x) .
\end{aligned}
$$

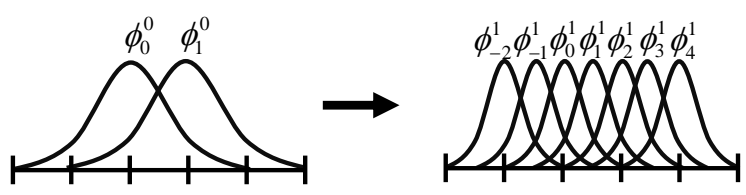

Fig. 14. Refinement of cubic B-splines.

To compute the approximation $P^{l}(x)$ at $x \in e_{l}$, we need the meshes from all samples $x_{k} \in \overline{\mathcal{B}}\left(e_{l}\right)$ to be represented in the canonical format of $e_{l}$. If $x_{k} \in \overline{\mathcal{B}}\left(e_{l}\right) \backslash \mathcal{B}\left(e_{l}\right)$, then $x_{k} \in \mathcal{B}\left(e_{\bar{l}}\right)$, where $e_{\bar{l}}$ is adjacent to $e_{l}$. Let $x_{\bar{k}}$ be a sample in $\mathcal{B}\left(e_{\bar{l}}\right) \cap \mathcal{B}\left(e_{l}\right)$ (see Figure 15). Since $p_{\bar{k}}^{l}$ and $p_{\bar{k}}^{\bar{l}}$ are stored during our pre-computational phase and have the same geometry, we can compute a mapping $F: p_{\bar{k}}^{\bar{l}} \rightarrow p_{\bar{k}}^{l}$ using barycentric coordinates and then apply it to $p_{k}^{\bar{l}}$ to obtain $p_{k}^{l}$. Locality ensures that this mapping only has to be done once for each sample guaranteeing that errors do not accumulate.

\section{HOMEOMORPHIC MAPPING}

In this section we discuss how we define a homeomorphic map that allows for representing a mesh $p_{A}$ in the canonical format of a given element, $p^{l}$. The resulting mesh $p_{A}^{l}$ should have the same geometry as $p_{A}$ and the same combinatorics as the canonical reference $p_{l}$ (see 


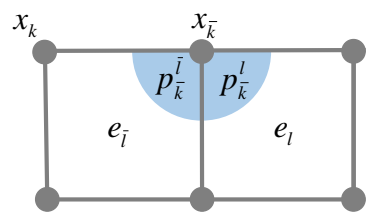

Fig. 15. The mapping $F: p_{\bar{k}}^{\bar{l}} \rightarrow p_{\bar{k}}^{l}$ is used to obtain $p_{k}^{l}$ for $x_{k} \in \overline{\mathcal{B}}\left(e_{l}\right) \backslash$ $\mathcal{B}\left(e_{l}\right)$.

Figure 19). To this end, we need to establish a dense correspondence between two meshes. Surface correspondence on meshes has been extensively studied in previous work [Van Kaick et al. 2011]. Our problem is distinct because we can take advantage of the CAD referencing methods to establish partial correspondence. As we will show in this section, this correspondence is not necessarily complete since the topology of the internal CAD representations (B-rep) may differ even if the geometry varies smoothly. We therefore propose an algorithm that combines $\mathrm{CAD}$ data analysis with mesh surface correspondence algorithms from previous work.

\subsection{Motivation}

As previously discussed, CAD systems use B-reps to represent solid models, which are generated by computing a list of features. A referencing scheme is used to determine the parts of the model to which features are applied, allowing these to be correctly regenerated if the model is modified. It is very common that parameter changes affect the topology of the B-rep, even in cases in which the geometry varies smoothly. Because references depend on the feature history, not the B-rep itself, they are robust to topological changes that do not affect the referenced element. An example of this is shown in Figure 16.
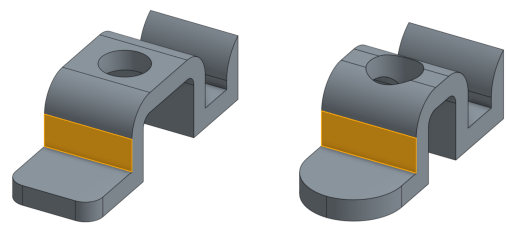

Fig. 16. Example of referencing in Onshape. The user applies a feature to a given face which is highlighted (left), and then changes parameter making other faces and edges of the model merge or split (right). Onshape's referencing scheme guarantees that the feature will still be applied to the correct face even after these changes are made.

The CAD system's API can be used to index each topological entity of the B-rep (faces, edges and vertices) from their internal referencing scheme. However, because faces can merge or split, we cannot generate a mapping simply from the correspondence between each face. In order to ensure that our method is robust to topological changes on the B-rep, we propose using the information from the CAD system to establish sparse correspondences on the surface of the shapes. This is then used to establish a dense correspondence map on the shape boundaries. Following the approach of previous work ([Aigerman et al. 2014, 2015a; Kraevoy and Sheffer 2004; Lee et al. 1999; Praun et al. 2001; Schreiner et al. 2004] to name a few) we use this sparse correspondence to split both source and target meshes into a common patch layout and compute the parameterization for each patch. Finally, we compute the volumetric mesh,
$p_{A}^{l}$, by propagating the boundary distortion map to all tetrahedrons in the interior.

\subsection{Algorithm}

$C A D$ Referencing. When sampling a point in the parametric domain $x_{k}$, we use a CAD system's API to update the parameters $x_{k}$, evaluate the model (i.e., recompute the feature list) and export the mesh $p_{k}$. Further, we use the API to query for a set of control variables that we define on the surface of the model in order to establish correspondences. Our control variables are a set of points with identifiers (IDs) and a set of paths between points. These control variables are set individually for each parameter configuration. We establish correspondences only when control variables with the same ID exist in both source and target meshes, addressing the case of varying B-rep topologies.

To generate these control variables, we designed a feature that references all of the vertices and edges of the solid model and tags each of these entities with a unique index. We add this feature to the end of the feature history. The reason we cannot simply use this index as the ID for each vertex and edge is that, though some references will break when entities vanish, others will be preserved even if the entities undergo topological changes. The referencing in CAD systems is designed this way in order to conserve certain operations even when the entities to which they are applied merge or split (see Figure 17). Though this is very powerful for CAD design, it is problematic for our application, since using these indices directly would allow us to create correspondences between vertices that collapse to a point or between two edges that are adjacent in one model and edges that are not adjacent in the other. To ensure a smooth surface mapping, these correspondences must be avoided since they will lead to shrinking the triangles in between to zero.
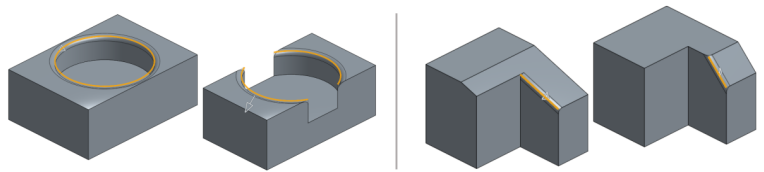

Fig. 17. Two examples of a fillet feature applied to edges. After this feature is defined, parameter changes on earlier features in the feature list split the edges into two parts. The way the edge references are handled in order to re-generate the fillet depends on the feature history, not the geometry. On the model on the left, the CAD system applies the fillet to both edges generated by the split; on the model on the right, the fillet is applied to only one of the edges.

To address this problem, we establish correspondences only between elements of the B-rep which undergo no topological changes. To this end, we define the ID for each vertex as the vertex index and a (sorted) vector of indices of adjacent edges. Analogously, we define the ID for each edge as the edge index and a (sorted) vector of indices of adjacent vertices. This simple scheme is sufficient to ensure that matching IDs exist only between elements with matching topologies.

Once IDs are established for every vertex and edge, control points are then set by computing the position of each referenced vertex and sampling each referenced edge. Sampling on edges is easily performed by the geometric kernel using the API. Though we would like the number of samples per edge to be proportional to the edge 
length, we require similar sampling on source and target meshes in order to establish correspondences. Since edge sizes can vary significantly when parameters are changed, we sample each edge by hierarchical subdivision until the length of each segment is smaller than a given threshold, and generate IDs for each sample according to this subdivision. In addition to the control points, our API call also returns a list of paths that connect pairs of points that are consecutive along an edge.

Surface Mapping. The common patch layout defines graphs on both source and target meshes. We use the set of control points with matching IDs to define the nodes on this graph. Edges on this graph define paths on the source and target meshes connecting these vertices. In the first stage, we use the paths extracted from the CAD system to create edges in this graph (see second row of Figure 18).

We then add edges to the patch layout, following the approach of previous work [Kraevoy and Sheffer 2004]. To this end, we first compute a list of candidate edges by computing shortest paths between every pair of control points in both source and target meshes and sorting this candidate list by the sum of the lengths of the source and target paths. These paths are constrained to (a) not intersect each other, (b) maintain the cyclical order, and (c) not place corresponding control points in two different patches. We use the candidate list to incrementally add edges to the patch layout. Every time a new edge is added, the candidate list is updated to ensure the constraints are satisfied.

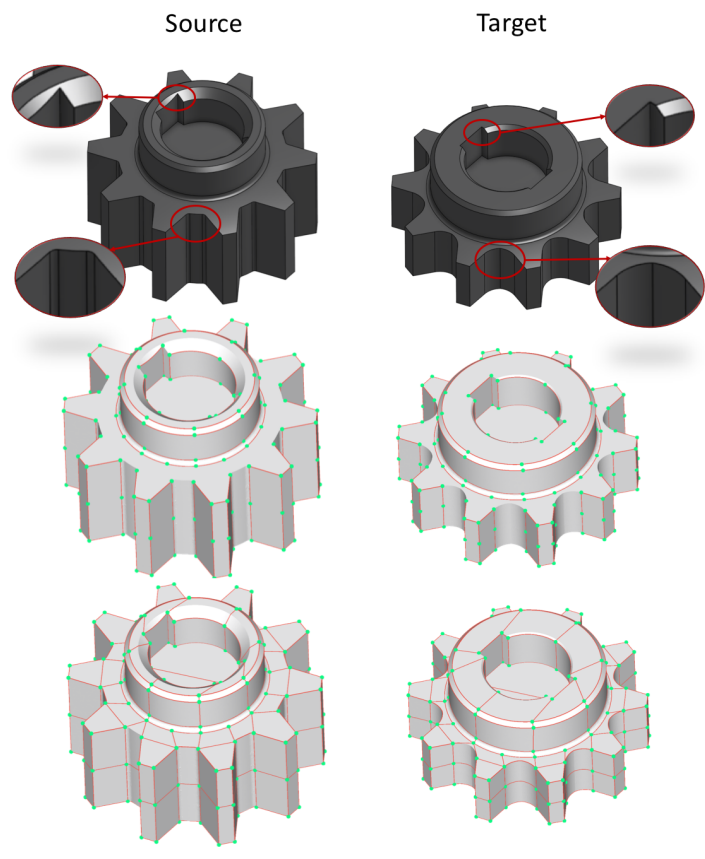

Fig. 18. Common patch layout given a source and target geometry. Top to bottom: B-rep with highlighted topological changes, paths extracted from $\mathrm{CAD}$, complete patch layout.

Edges are selected first to ensure the graph is connected and then to ensure that every patch is simple. Finally, more edges are added to further subdivide large patches for better parametrization.
In this step, however, we add an edge only if the shortest path length is significantly smaller than the path that connects these two points on the patch layout. Adding this restriction is necessary for our application because details such as small fillets or holes can generate a large number of control points that are very close to each other. Finally, we refine paths to geodesic paths. Using geodesic paths smooths the boundary, making the paths on the target and source meshes more consistent. This is done only after the paths are selected because it is a time-consuming step. Finally, we construct a mapping between each path pair using a mean-value parametrization technique [Floater 2003] to map each patch onto a disk and then composing the two maps to establish a mapping from the source to target patches.

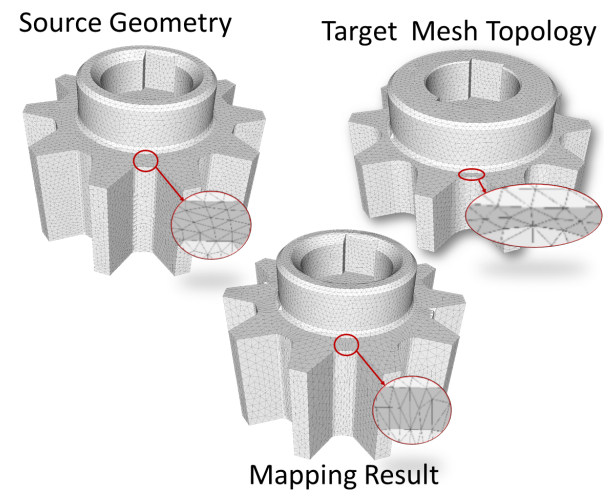

Fig. 19. Mapping result. Given a source geometry and a target mesh topology the mapping outputs a mesh with the source geometry and target topology.

Interior mapping. Since, in our problem, meshes are reasonably similar, we can map the surface mapping to the interior by solving a linear elastic FEM problem. We use the surface map as the Dirichlet boundary conditions for the FEM simulation, and compute the volumetric deformation for the interior map.

Boundary Conditions. In addition to mapping the shapes, we also need to establish consistent boundary conditions for our simulations. Consider, for example, a chair model in which the force is applied to the seat, which can vary in length and width. In order to simulate this result, we need to know which mesh vertices correspond to the seat as the mesh varies. We address this problem by developing a boundary condition feature to be added at the end of a solid model's feature list. These features take as inputs the faces of the model and the information of the boundary condition type (forces, temperature, etc). Similar to the discussion above, we use the CAD referencing scheme to find the mesh regions that correspond to the selected faces. This allows us to establish consistent boundary regions during interpolation.

\section{RESULTS AND DISCUSSION}

While our method can be applied to any CAD system, we have implemented the discussed techniques using Onshape's API. We discuss examples for exploration and optimization applications. 


\subsection{Shape Exploration}

Our precomputed data and interpolation scheme allow visualization of geometry variations in real time. Our tool can further display to the user physical properties that depend on the geometry and can be computed at interactive rates. In our experiments, we evaluate mass, surface area, drag, and moment of inertia. Other physical properties that are defined over the mesh and require expensive computations can be precomputed for every sample and interpolated with our scheme. Different applications require analysis of different properties. When constructing the K-d tree, we define the error metric for determining if an element should be refined by either comparing the geometry (with Hausdorff distances) or the error on the precomputed simulation in applications where these properties are added.

Figure 1 and the first four rows of Figure 20 show examples with precomputed stress analysis. Figure 1 shows a wrench that is constrained to be fixed in the faces which are in contact with a bolt head and has a force pulling down on the handle. The result displays the moment of inertia, mass and stress distribution as three parameters vary: the head radius, the handle length and the fillet connecting this two parts.

The first row of Figure 20 shows a rocker arm on a bike that is fixed to the suspension component and has a force from the rear wheel. The four parameters include directions and length of the inner holes, thickness of the part and fillet radius on the border. Typically, an engineer will try to minimize the weight and drag of a model like this while keeping stress below a certain threshold. Our interface displays all properties in real time. This model highlights an advantage of this visualization tool over a direct optimization since both the thickness and the hole size affect all variables and this tool allows users to choose from the multiple configurations that meet the design criteria.

The second row shows a chair with precomputed stress analysis which results from a force on the seat. Our mapping of boundary conditions allows the force to be uniformly applied on the surface of the seat across parameter variations which affect its dimensions. In this example a total of six parameters can be exposed since a chair does not need to interface with other models in an assembly, allowing greater variability.

The third row is a camera mount, which has fixed sizes and a downwards force from the weight of the camera. The engineer aimed at minimizing both the mass and the deformation while making the stress distribution as even as possible. Using our tool to view all these properties at interactive rates, the engineer can find an optimal solution without needing to come up with reasonable weights for a composed objective function to be used in optimization. The holes were designed as circular patches with varying radii which can be fabricated with punching, which is far less expensive than milling, illustrating how CAD data can be applied for optimization with fabrication constraints.

The fourth row is a swing castle where the dimensions of the door and windows are parametrized. Since this model requires expensive coating, surface area is displayed along with the pre-computed stress analysis. In this example, the direction of the force on the bar varies as the child swings. This direction is treated as a third parameter in our interface allowing users to visualize the stress distribution as both the geometry and the force vary.

The fifth row shows an example of a toy airplane for which the air pressure distribution was precomputed using a compressible flow solver. Our interactive tool displays this property together with moment of inertia and drag from a frontal wind which are directly computed from the geometry at interactive rates.

Finally, the sixth row shows an example where thermal expansion is coupled with the stress from the brake hub. The heat source is uniform on the inner walls while the forces from the brake are directional. We measure the stress that results from both the directional forces and the heat deformations. We store the pre-computed heat distribution, stress, and deformation. The engineer can explore the shape and size of the inner holes to normalize the stress distribution and also vary the thickness of the rim. We observe that as the latter increases, the deformation on the rim becomes more uniform since the impact of the heat outweighs the directional forces.

Table 1 describes the number of parameters, levels of the K-d tree, total sampled nodes, average mesh size for each model, and overall number of meshes stored. For precomputation, the time to evaluate each instance (specific parameter values) is the latency of the CAD evaluation and physical simulation. In the examples, geometry evaluation for each instance took about one minute, including B-rep regeneration from Onshape and tetrahedralization. Simulation times range from 5 to 10 minutes for stress analysis, and 2 to 3 minutes for thermoelastic and air pressure distribution. Our interactive viewer updates all examples in real time.

Table 1. The number of parameters (K), levels of the K-d tree, total sampled nodes, average mesh size (in number of tetrahedrons), and total storage (in number of stored meshes) for each model.

\begin{tabular}{|c|ccccc|}
\hline model & K & levels & samples & mesh size & storage \\
\hline \hline wrench & 3 & 5 & 49 & $386 \mathrm{k}$ & 112 \\
chair & 6 & 7 & 777 & $61 \mathrm{k}$ & 4224 \\
bike frame & 4 & 6 & 103 & $288 \mathrm{k}$ & 304 \\
camera mount & 5 & 6 & 259 & $291 \mathrm{k}$ & 1056 \\
swing & 3 & 5 & 58 & $211 \mathrm{k}$ & 144 \\
plane & 4 & 5 & 148 & $429 \mathrm{k}$ & 432 \\
drum brake & 3 & 6 & 72 & $356 \mathrm{k}$ & 208 \\
\hline
\end{tabular}

Table 2 shows the approximation error for both geometry and elastic FEM simulation for each model. This is measured by sampling 10 random points in the domain and comparing the evaluated shapes and physical properties with our approximation. We observe that while geometry reconstruction is quite accurate, physical properties do not interpolate as well since they are highly non-linear. Still, we argue that it is accurate enough for a visualization cycle of gaining intuition since it clearly highlights how each parameter affects the physical properties of the model and which ones are the most relevant. When high accuracy is crucial, designers can use this tool to select optimal configurations and then run simulations at a second stage for a small number of selected parameters. We argue, however, that in most practical scenarios this second stage is not necessary because the approximation error is reasonable given engineering safety factors. 

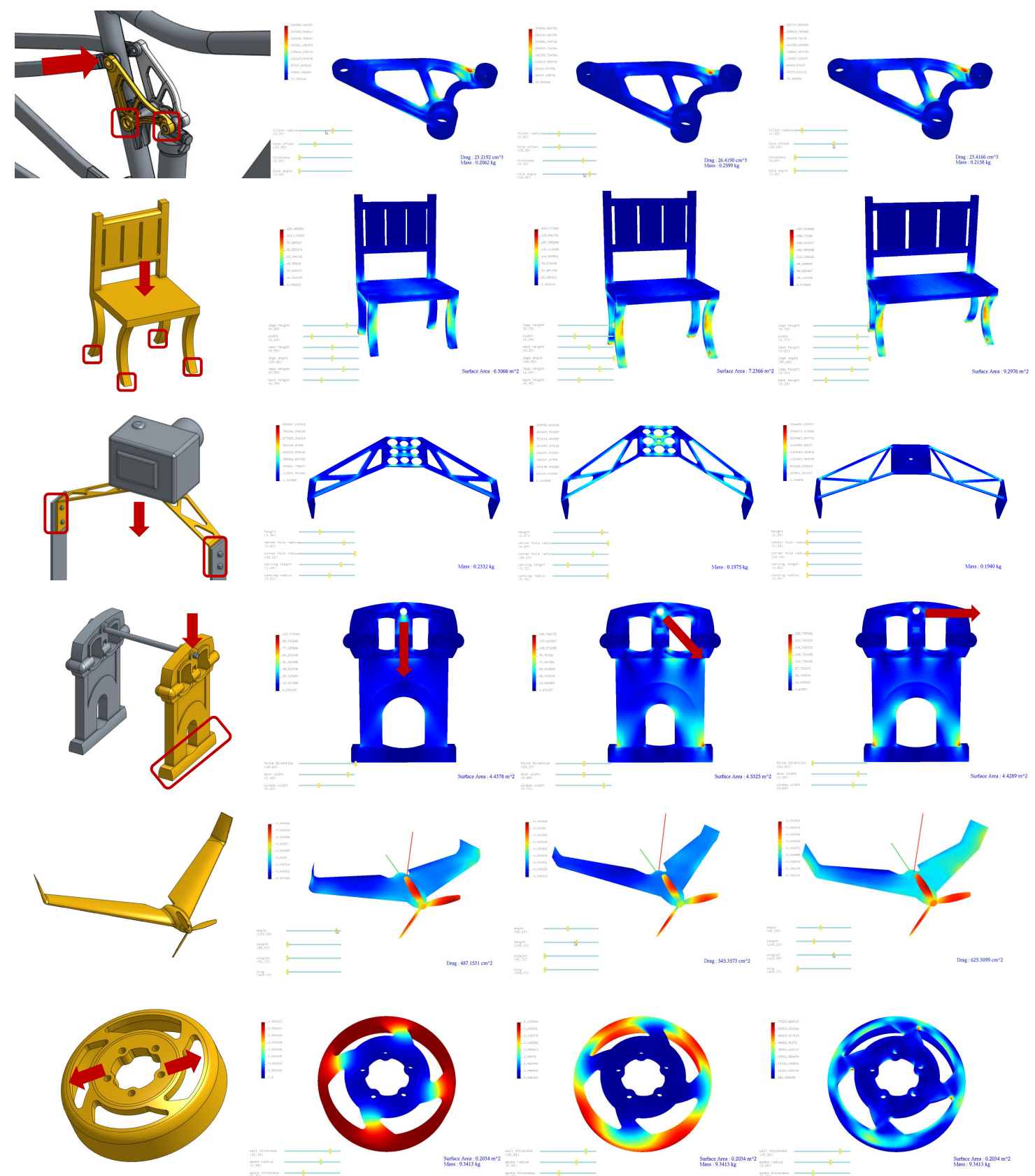

Fig. 20. Examples of interactive visualization. On the left we show each model in red boxes and the regions with fixed boundary conditions and forces with red arrows. On the right we show results from our visualization interface. As the user varying the parameters, geometry is updated is real time. The top four rows show pre-computed stress analysis. On the forth row the force direction also varies and is illustrated with a red arrow. The fifth row shows a result with fluid simulation. The last row shows a results with thermoelastic simulation where the colors display (from left to right) heat distribution, deformation and stress.

When engineers design a part, they typically use simulation at multiple configurations in order to optimize physical properties. Since each simulation takes a long time, they use their training and expertize to pick certain configurations for testing and then to tweak the parameters based on these results. This process requires expert knowledge because each simulation takes a very long time and the more experience they have the fewer iterations are required [Foshey et al. 2017]. For these reasons, interactive tools such as this one have the potential to not only facilitate the design process for engineers but also lower the barrier for novice users. In addition, it can be used to help less experienced engineers gain this intuition since it is a very informative way of illustrating how geometry impacts physical properties. 
Table 2. Relative approximation error on geometry and elastic FEM for all example models.

\begin{tabular}{|c|cccc|}
\hline model & $\begin{array}{c}\text { geometry } \\
\max \end{array}$ & $\begin{array}{c}\text { geometry } \\
99 \%\end{array}$ & $\begin{array}{c}\text { FEM } \\
\max \end{array}$ & $\begin{array}{c}\text { FEM } \\
99 \%\end{array}$ \\
\hline \hline wrench & 0.0028 & 0.0004 & 0.2138 & 0.0393 \\
chair & 0.0243 & 0.0022 & 0.3756 & 0.1130 \\
bike frame & 0.0112 & 0.0003 & 0.1403 & 0.0281 \\
camera mount & 0.0009 & 0.0002 & 0.4107 & 0.1861 \\
swing & 0.0013 & 0.0002 & 0.4750 & 0.2042 \\
plane & 0.0162 & 0.0055 & - & - \\
drum brake & 0.0016 & 0.0006 & 0.3095 & 0.0490 \\
\hline
\end{tabular}

\subsection{Shape Optimization}

We also show how our method can be used for shape optimization. For this application we use cubic B-splines, which guarantee smooth interpolations. Using Equation 1, we can define the derivatives of our approximation as

$$
P^{\prime}(x)=\sum_{k} p_{k} \psi_{k}^{\prime}(x)
$$

which can be expressed analytically since $\psi_{k}(x)$ is a sum of weighted B-splines. We use this to drive a gradient-based algorithm for optimization over objective functions defined on the mesh. We use an interior point method and IpOpt for implementation.

Figure 21 shows an example of an optimization result where the objective function is defined as the integral of the stress over the volume. This arbor press has five parameters, two that define the dimensions and three that are structural (fillet radius, side thickness and hole size). Since different applications require different dimensions, companies will typically sell a range of similar shapes with varying heights and widths. This shows a typical use case for our method when a shape can be pre-computed once and then optimized for different use cases. The figure shows results for two configurations where heights and widths are fixed and the structural variables are optimized.
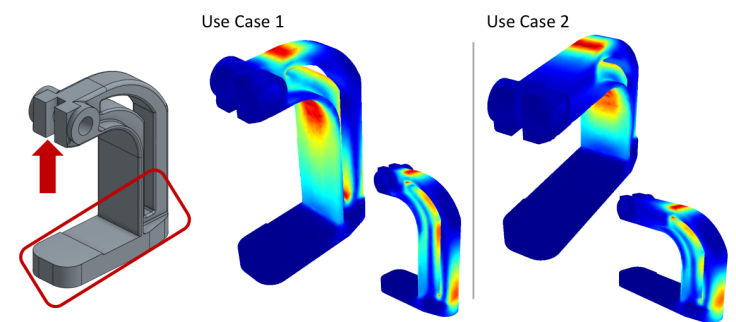

Fig. 21. Result of shape optimization. Stress is minimized on an arbor press for two different use cases, a tall one is shown on the right and a short one on the left.

\subsection{Limitations and Future Work}

While the main technical contributions of our work involve interpolation, this cannot be applied for discrete parameters or topological changes. In such scenarios, our method can still be used to interpolate the continuous variations between discrete changes of parameters or topology. In the future it would be interesting to explore this technique in tools that combine discrete and continuous exploration such as the one proposed by Bao at el. [2013].
Another limitation of our precomputation scheme is that it does not scale well with the number of parameters. In practice, however, this is not a problem because the number of parameters that can be exposed tend to be small due to large number of constraints driven by manufacturing considerations or the need to interface with other models [Baran 2017; Schulz et al. 2017; Shugrina et al. 2015].

Storing $2^{K}$ meshes for every sample is arguably another limitation of our approach. In our implementation, this was not a problem even in the case of high resolution meshes. In the future, however, it could be interesting to incorporate compression schemes on the meshes [Alliez and Gotsman 2005]. Additionally the physical properties computed can also be compressed, as is done in this work for corner vibration modes [Langlois et al. 2014].

Since the interface with the CAD system allows detailed control variables that highlight the sharp features of the model and since shape variations from parameter changes are relatively small, we were able to extract satisfactory results with simple surface correspondence algorithms. In the future, it would be interesting to combine our method for extracting control variables from CAD with more recent approaches such as [Aigerman et al. 2015b], which would guarantee more smoothness on patch boarders. It would also be interesting to experiment with mesh morphing algorithms that are not linear [Alexa et al. 2000].

While in the examples shown we use precomputation to incorporate physics simulations, an alternative is to use sensitivity analysis [Umetani et al. 2011] and reduced models [Chen et al. 2016] for real-time physics exploration. These approaches also require real time evaluation of geometry and derivatives with respect to parameter changes that are provided by our methods. These techniques been proven successful for other types of parametric shapes, and it would be interesting to couple them with our method, extending them to applications with CAD data.

\section{CONCLUSION}

In this work we propose a precomputation approach to allow interactive exploration and optimization of CAD data. Our main technical contributions is an interpolation scheme for adaptive grids that simultaneously guarantees continuity/smoothness and locality. This is a general interpolation algorithm that we hope will find many applications

We show examples of how our method can be coupled with different physics simulations to allow designers to interactively or automatically optimize shapes for different objectives. Since shape regeneration from $\mathrm{CAD}$ parameters and accurate evaluations of their physical properties are inherently slow, the existing workflow for optimizing parameters is not only time consuming but also requires expertise since only a small set of variations can be tested in a reasonable amount of time. In this context, our tool not only helps experts to efficiently search the design space but also helps bring down the design barrier to casual users.

We argue that $\mathrm{CAD}$ data is an exciting data collection not only because of its size and influence but also because the parameters in this representation expose engineering design intent including manufacturing considerations. Because this work allows real-time geometry generation from parameter settings and continuous derivatives, it paves the way to applying this parametric data to works that use 
exploration and optimization of parametric shapes. We hope that the techniques we present in this work will allow $\mathrm{CAD}$ data to be applied to the plethora of the existing and upcoming research works in the growing field of fabrication-oriented design.

\section{ACKNOWLEDGMENTS}

The authors would like to thank Ilya Baran for helpful suggestions and discussions, Michael Foshey, Nicholas Bandiera and Javier Ramos for discussions and for designing the models in the examples, and the team at Onshape for support with the API.

\section{REFERENCES}

Aseem Agarwala. 2007. Efficient Gradient-domain Compositing Using Quadtrees. In Siggraph 2007. ACM.

Noam Aigerman, Roi Poranne, and Yaron Lipman. 2014. Lifted bijections for low distortion surface mappings. ACM Trans. Graph. 33, 4 (2014), 69.

Noam Aigerman, Roi Poranne, and Yaron Lipman. 2015a. Seamless surface mappings. ACM Trans. on Graph. (TOG) 34, 4 (2015), 72.

Noam Aigerman, Roi Poranne, and Yaron Lipman. 2015b. Seamless Surface Mappings. ACM Trans. Graph. 34, 4 (July 2015), 72:1-72:13.

Marc Alexa, Daniel Cohen-Or, and David Levin. 2000. As-rigid-as-possible shape interpolation. In Siggraph 2000. ACM, 157-164.

Pierre Alliez and Craig Gotsman. 2005. Recent advances in compression of 3D meshes. In Advances in multiresolution for geometric modelling. Springer, 3-26.

Mehdi Baba-Ali, David Marcheix, and Xavier Skapin. 2009. A method to improve matching process by shape characteristics in parametric systems. Computer-Aided Design and Applications 6, 3 (2009), 341-350.

Moritz Bächer, Stelian Coros, and Bernhard Thomaszewski. 2015. LinkEdit: Interactive Linkage Editing Using Symbolic Kinematics. ACM Trans. Graph. 34, 4 (July 2015), 99:1-99:8.

Fan Bao, Dong-Ming Yan, Niloy J. Mitra, and Peter Wonka. 2013. Generating and Exploring Good Building Layouts. ACM Trans. Graph. 32, 4 (July 2013), 122:1122:10.

Ilya Baran. 2017. Onshape Inc. Personal Communication. (2017).

David Benson and Joel Davis. 2002. Octree textures. ACM Transactions on Graphics 21, 3 (2002), 785-790.

Gaurav Bharaj, David I. W. Levin, James Tompkin, Yun Fei, Hanspeter Pfister, Wojciech Matusik, and Changxi Zheng. 2015. Computational Design of Metallophone Contact Sounds. ACM Trans. Graph. 34, 6 (Oct. 2015), 223:1-223:13.

Rafael Bidarra and Willem F Bronsvoort. 2000. Semantic feature modelling. ComputerAided Design 32, 3 (2000), 201-225.

Rafael Bidarra, Paulos J Nyirenda, and Willem F Bronsvoort. 2005. A feature-based solution to the persistent naming problem. Computer-Aided Design and Applications 2, 1-4 (2005), 517-526.

Martin Bokeloh, Michael Wand, Hans-Peter Seidel, and Vladlen Koltun. 2012. An Algebraic Model for Parameterized Shape Editing. ACM Trans. Graph. 31, 4 (July 2012), 78:1-78:10

Xiang Chen, Changxi Zheng, and Kun Zhou. 2016. Example-Based Subspace Stress Analysis for Interactive Shape Design. IEEE Transactions on Visualization and Computer Graphics (2016).

Tao Du, Adriana Schulz, Bo Zhu, Bernd Bickel, and Wojciech Matusik. 2016. Computational Multicopter Design. ACM Trans. Graph. 35, 6 (Nov. 2016), 227:1-227:10.

Gerald E Farin, Josef Hoschek, and Myung-Soo Kim. 2002. Handbook of computer aided geometric design. Elsevier.

Michael S Floater. 2003. Mean value coordinates. Computer aided geometric design 20, 1 (2003), 19-27.

Michael S Floater. 2015. Generalized barycentric coordinates and applications. Acta Numerica 24 (2015), 161-214.

David R. Forsey and Richard H. Bartels. 1988. Hierarchical B-spline Refinement. In Siggraph 1988. ACM, 205-212.

Michael Foshey, Nicholas Bandiera, and Javier Ramos. 2017. Mechanical Engineers at MIT. Personal Communication. (2017).

Eitan Grinspun, Petr Krysl, and Peter Schröder. 2002. CHARMS: A Simple Framework for Adaptive Simulation. ACM Trans. Graph. 21, 3 (July 2002), 281-290.

Alec Jacobson, Ilya Baran, Jovan Popovic, and Olga Sorkine. 2011. Bounded biharmonic weights for real-time deformation. ACM Trans. Graph. 30, 4 (2011), 78.

Leif Kobbelt, Marc Stamminger, and Hans-Peter Seidel. 1997. Using Subdivision on Hierarchical Data to Reconstruct Radiosity Distribution. Computer Graphics Forum (1997).

Yuki Koyama, Shinjiro Sueda, Emma Steinhardt, Takeo Igarashi, Ariel Shamir, and Wojciech Matusik. 2015. AutoConnect: Computational Design of 3D-printable Connectors. ACM Trans. Graph. 34, 6 (Oct. 2015), 231:1-231:11.
Vladislav Kraevoy and Alla Sheffer. 2004. Cross-parameterization and Compatible Remeshing of 3D Models. In Siggraph 2004. ACM, 861-869.

Timothy R Langlois, Steven S An, Kelvin K Jin, and Doug L James. 2014. Eigenmode compression for modal sound models. ACM Trans. Graph. 33, 4 (2014), 40.

Aaron WF Lee, David Dobkin, Wim Sweldens, and Peter Schröder. 1999. Multiresolution mesh morphing. In Siggraph 1999. ACM, 343-350.

Seungyong Lee, George Wolberg, and Sung Yong Shin. 1997. Scattered data interpolation with multilevel B-splines. IEEE transactions on visualization and computer graphics 3, 3 (1997), 228-244.

Frank Losasso, Frédéric Gibou, and Ron Fedkiw. 2004. Simulating Water and Smoke with an Octree Data Structure. ACM Trans. Graph. 23, 3 (Aug. 2004), 457-462.

Vittorio Megaro, Bernhard Thomaszewski, Maurizio Nitti, Otmar Hilliges, Markus Gross, and Stelian Coros. 2015. Interactive Design of 3D-printable Robotic Creatures. ACM Trans. Graph. 34, 6 (Oct. 2015).

Przemyslaw Musialski, Thomas Auzinger, Michael Birsak, Michael Wimmer, and Leif Kobbelt. 2015. Reduced-order Shape Optimization Using Offset Surfaces. ACM Trans. Graph. 34, 4 (July 2015).

Przemyslaw Musialski, Christian Hafner, Florian Rist, Michael Birsak, Michael Wimmer, and Leif Kobbelt. 2016. Non-linear Shape Optimization Using Local Subspace Projections. ACM Trans. Graph. 35, 4 (July 2016).

Emil Praun, Wim Sweldens, and Peter Schröder. 2001. Consistent mesh parameterizations. In Siggraph 2001. ACM, 179-184.

Romain Prévost, Emily Whiting, Sylvain Lefebvre, and Olga Sorkine-Hornung. 2013. Make It Stand: Balancing Shapes for 3D Fabrication. ACM Trans. Graph. 32, 4 (July 2013), 81:1-81:10.

John Schreiner, Arul Asirvatham, Emil Praun, and Hugues Hoppe. 2004. Inter-surface mapping. In ACM Trans. Graph., Vol. 23. ACM.

Adriana Schulz, Ariel Shamir, Ilya Baran, David I. W. Levin, Pitchaya Sitthi-Amorn, and Wojciech Matusik. 2017. Retrieval on Parametric Shape Collections. ACM Trans. Graph. 36, 1 (Jan. 2017), 11:1-11:14.

Adriana Schulz, Ariel Shamir, David I. W. Levin, Pitchaya Sitthi-amorn, and Wojciech Matusik. 2014. Design and Fabrication by Example. ACM Trans. Graph. 33, 4 (July 2014), 62:1-62:11.

Thomas W. Sederberg, Jianmin Zheng, Almaz Bakenov, and Ahmad Nasri. 2003. Tsplines and T-NURCCs. ACM Trans. Graph. 22, 3 (July 2003).

Rajsekhar Setaluri, Mridul Aanjaneya, Sean Bauer, and Eftychios Sifakis. 2014. SPGrid: A Sparse Paged Grid Structure Applied to Adaptive Smoke Simulation. ACM Trans. Graph. 33, 6 (Nov. 2014).

Maria Shugrina, Ariel Shamir, and Wojciech Matusik. 2015. Fab Forms: Customizable Objects for Fabrication with Validity and Geometry Caching. ACM Trans. Graph. 34, 4 (July 2015), 100:1-100:12.

Mélina Skouras, Stelian Coros, Eitan Grinspun, and Bernhard Thomaszewski. 2015 Interactive Surface Design with Interlocking Elements. ACM Trans. Graph. 34, 6 (Oct. 2015).

Ian Stroud. 2006. Boundary representation modelling techniques. Springer Science \& Business Media.

N Sukumar and EA Malsch. 2006. Recent advances in the construction of polygonal finite element interpolants. Archives of Computational Methods in Engineering 13, 1 (2006), 129-163.

Nobuyuki Umetani, Takeo Igarashi, and Niloy J. Mitra. 2012. Guided Exploration of Physically Valid Shapes for Furniture Design. ACM Trans. Graph. 31, 4 (2012).

Nobuyuki Umetani, Danny M. Kaufman, Takeo Igarashi, and Eitan Grinspun. 2011. Sensitive Couture for Interactive Garment Modeling and Editing. ACM Trans. Graph. 30, 4 (July 2011), 90:1-90:12.

Nobuyuki Umetani, Yuki Koyama, Ryan Schmidt, and Takeo Igarashi. 2014. Pteromys: Interactive Design and Optimization of Free-formed Free-flight Model Airplanes. ACM Trans. Graph. 33, 4 (July 2014), 65:1-65:10.

Oliver Van Kaick, Hao Zhang, Ghassan Hamarneh, and Daniel Cohen-Or. 2011. A survey on shape correspondence. In Computer Graphics Forum, Vol. 30. Wiley Online Library, 1681-1707.

Emily Whiting, Hijung Shin, Robert Wang, John Ochsendorf, and Frédo Durand. 2012 Structural Optimization of 3D Masonry Buildings. ACM Trans. Graph. 31, 6 (2012), 159:1-159:11.

Kun Xu, Yong Li, Tao Ju, Shi-Min Hu, and Tian-Qiang Liu. 2009. Efficient Affinity-based Edit Propagation Using K-D Tree. In Siggraph Asia 2009. ACM, 118:1-118:6.

Youyi Zheng, Hongbo Fu, Daniel Cohen-Or, Oscar Kin-Chung Au, and Chiew-Lan Tai. 2011. Component-wise Controllers for Structure-Preserving Shape Manipulation. In Computer Graphics Forum, Vol. 30. Wiley Online Library, 563-572.

Denis Zorin and Peter Schroder. 2000. Subdivision for Modeling and Animation. In Siggraph 2000 Courses. ACM. 